\title{
Pulsed homodyne measurements of femtosecond squeezed pulses generated by single-pass parametric deamplification
}

\author{
Jérôme Wenger, Rosa Tualle-Brouri and Philippe Grangier \\ Laboratoire Charles Fabry de l'Institut d'Optique, CNRS UMR 8501, F-91403 Orsay, France.
}

\begin{abstract}
A new scheme is described for pulsed squeezed light generation using femtosecond pulses parametrically deamplified through a single pass in a thin $(100 \mu \mathrm{m})$ potassium niobate $\mathrm{KNbO}_{3}$ crystal, with a significant deamplification of about $-3 \mathrm{~dB}$. The quantum noise of each individual pulse is registered in the time domain using a single-shot homodyne detection operated with femtosecond pulses and the best squeezed quadrature variance was measured to be $1.87 \mathrm{~dB}$ below the shot noise level. Such a scheme provides the basic ressource for time-resolved quantum communication protocols.
\end{abstract}

PACS numbers: 03.67.-a, 42.50.Dv, 03.65.Wj

In the presently very active field of quantum information processing using continuous variables [1], the generation and detection of squeezed states of light is a topic of considerable interest, since these states may be used as the direct ressource for efficient protocols in quantum cryptography, entanglement generation, quantum teleportation or dense coding. Moreover, squeezing appears as a fundamental ressource for universal quantum computation with continuous variables.

To generate squeezed states, the use of ultrashort pulses, with their high peak power and their potential for pulse shaping, has attracted lots of attention since the landmark pulsed squeezing experiment of Slusher et al [2]. Among many nonlinear interactions, the single-pass parametric amplifier appears as a relatively simple and efficient source of pulsed squeezed light [2, 3, 4, 5, 6, 7].

In this Letter, we describe a new scheme for pulsed squeezed light generation using 150 fs pulses parametrically deamplified through a single pass in a thin (100 $\mu \mathrm{m})$ potassium niobate $\left(\mathrm{KNbO}_{3}\right)$ crystal with a significant deamplification of about $-3 \mathrm{~dB}$. The femtosecond squeezed pulses are then sampled by a pulsed timeresolved homodyne detection, showing a quadrature variance reduced up to $1.87 \mathrm{~dB}$ below the shot noise level in good agreement with the measured classical deamplification $(0.53$ or $-2.76 \mathrm{~dB})$ and the homodyne measurement efficiency $(\eta=76 \%)$.

To our knowledge, this is the first time such a thin 100 $\mu \mathrm{m}$ long $\mathrm{KNbO}_{3}$ crystal is used with ultrashort pulses to perform second harmonic generation and parametric amplification. This crystal length allows for wide phasematching bandwidth and avoids the conditions of large group-velocity mismatch, contrary to the previously reported use of thick $\mathrm{KNbO}_{3}$ crystals [8, 99. Even for the short interaction length used here, $\mathrm{KNbO}_{3}$ proved to be suitable to our applications thanks to its high non linear coefficient (about $12 \mathrm{pm} / \mathrm{V}$ ) and non-critical phasematching.

Another fundamental point of our experiment is that all the processing is done in the time domain and not in the frequency domain, as it is often the case even for pulsed squeezing experiments [2]. The time resolved homodyne detection samples the quantum properties of

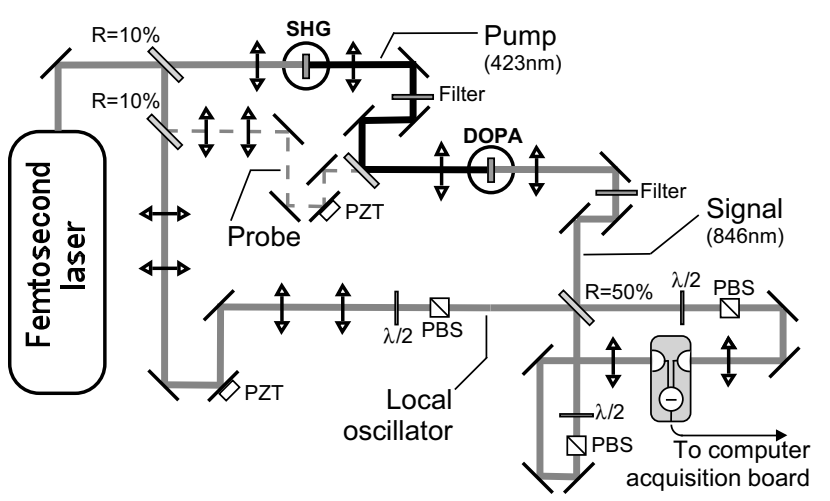

FIG. 1: Experimental setup

each individual incoming pulse and is directly sensitive to the pulse statistical distribution. It is thus very easy to analyse our experiment in terms of information transfers involved in quantum communication protocols 10 ]. Such single-shot homodyne detections have already been reported in the nanosecond 10 or picosecond 7, 11] domain, but to our knowledge there is no report of a pulsed homodyne detection operated with femtosecond pulses.

The experimental scheme is presented on Fig. [1 The initial pulses are obtained from a cavity-dumped titanium-sapphire laser (Tiger-CD, Time-Bandwidth Products), delivering nearly Fourier-transform limited pulses with a duration of $150 \mathrm{fs}$ centered at $846 \mathrm{~nm}$ (FWHM 5nm), with an energy up to $75 \mathrm{~nJ}$ at a pulse repetition rate of $790 \mathrm{kHz}$.

These pulses are focused near the center of an a-cut $100 \mu \mathrm{m}$ thick anti-reflection coated $\mathrm{KNbO}_{3}$ crystal (FEE $\mathrm{GmbH}$ ) in a lens arrangement to have a waist inside the crystal of about $16 \mu \mathrm{m}$. The crystal is set inside a small vacuum chamber and peltier-cooled down to about $-14^{\circ} \mathrm{C}$ to obtain non-critical (90 degrees) type-I phasematching for second harmonic generation (SHG) at 846 $\mathrm{nm}$. The best SHG efficiency obtained was of $32 \%$ (corrected from losses), with a typical value of about $28 \%$ (depending on the laser settings).

We have investigated the SHG conversion efficiency versus the fundamental power and versus the position 


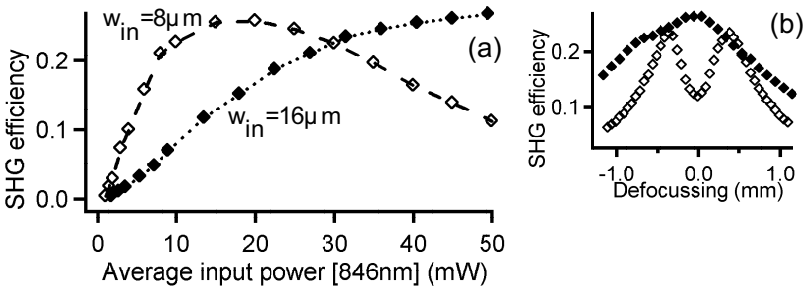

FIG. 2: (a) SHG efficiency versus average input power for an inside crystal waist of $8 \mu \mathrm{m}$ (empty diamonds) and $16 \mu \mathrm{m}$ (filled diamonds). (b) SHG efficiency when shifting the beam waist from the crystal center (defocussing) for an input power of $50 \mathrm{~mW}$.

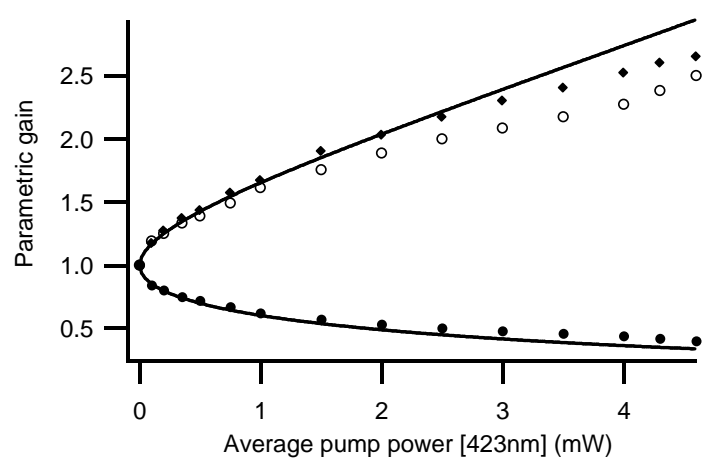

FIG. 3: Parametric gain versus average pump power at $423 \mathrm{~nm}$. Filled circles and diamonds correspond to deamplification and amplification, while empty circles mark the inverse of the deamplification gain. Solid lines corresponds to a fit according to plane wave theory.

of the focus inside the crystal (see Fig 2). With high input power, the SHG conversion efficiency decreases when the input beam is too tightly focused, as a dip in the conversion appears while varying the position of the focus around the center of the crystal. Studying $3 \mathrm{~mm}$ and $10 \mathrm{~mm}$ thick $\mathrm{KNbO}_{3}$ crystals, other teams also reported a decrease in the SHG efficiency for high input powers when tightly focusing $[\underline{8},[9]$, which they related to strong pump depletion combined with blue light induced infrared absorption 12] (BLIIRA). Due to the small interaction length of our experiment, BLIIRA appears here of less importance, as some other effects have to be taken into account in order to understand this optical damage : two-photon absorption 13] or charge carriers ionization may lead to local thermal heating of the material, which would result in a local change of the refractive index and destroy phase-matching. The buildup of a space-charge field would also affect phase-matching conditions through a photorefractive effect [14]. We are presently working on studying this phenomenon further.

A small fraction (1\%) of the fundamental beam is taken out to serve as a probe to study classical parametric amplification occurring in a similar $\mathrm{KNbO}_{3}$ crystal used in a single-pass type-I spatially degenerate configuration (DOPA). The relative phase between the probe and the blue pump determines the amplification or deamplification gain. These gains are recorded using direct detection of the probe beam averaged power on a photodiode. The best classical deamplification obtained was $0.40(-4.0$ $\mathrm{dB})$ with a corresponding amplification of $2.65(+4.2 \mathrm{~dB})$. Fig. 3 shows the classical gain versus the blue pump main power, together with the curve corresponding to planewave theory set to fit for pump powers below $0.5 \mathrm{~mW}$. Not surprisingly, for larger pump power the plane-wave fit do not overlap the experimental points, as plane-wave theory cannot account for the use of focused Gaussian beams and ultrashort pulses. The difference between amplification and the inverse of deamplification appears also more important at high pump powers. The phenomenon of gain-induced diffraction [15] is known to induce a lack of deamplification at high pump powers. Due to the Gaussian transverse dependence of the pump intensity, the portion of the probe beam closer to the propagation axis is more amplified than its wings, which distorts the probe phase front, degrades phase-matching and limits deamplification. Experimentally, we have optimised the overlap between the pump and the probe beam to get the best deamplification. We found that the best deamplification occurred when the probe waist was set to be about $\sqrt{2}$ smaller than the blue pump waist inside the DOPA crystal. This experimental optimisation appears as a compromise between small wavefront distorsion and spatial overlap between probe and pump.

The probe beam being blocked, the amplifier generates squeezed vacuum which is made to interfere with the local oscillator beam (LO) in a balanced homodyne detection setup. Achieving a good temporal and spatial mode matching between the squeezed vacuum and LO constitutes a major issue. To this end, the probe beam helped reaching an interference visibility of $93.5 \%$.

The homodyne detection is set to be directly sensitive to the pulse distribution in the time domain. For each incoming pulse, the fast acquisition board (National Instruments PCI-6111E) samples one value of the signal quadrature in phase with the local oscillator, allowing to directly construct the histograms presented below [7, 10, 11]. Such pulsed homodyning is technically much more challenging than frequency-resolved homodyning. Each arm of the detection has to be carefully balanced (with a typical rejection better than $10^{-4}$ ) even for ultralow frequency noises. By blocking the squeezed beam, the detection was checked to be shot-noise limited in the time domain, showing a linear dependence between LO power and the noise variance up to $2.510^{8}$ photons per pulse at a repetition rate of $790 \mathrm{kHz}$ and in the femtosecond regime. The electronic noise was low enough to ensure a ratio larger than $11 \mathrm{~dB}$ between shot noise and electronic noise variances.

Fig. 4 displays the recorded quadrature variance and the corresponding noise pulses while scanning the local 


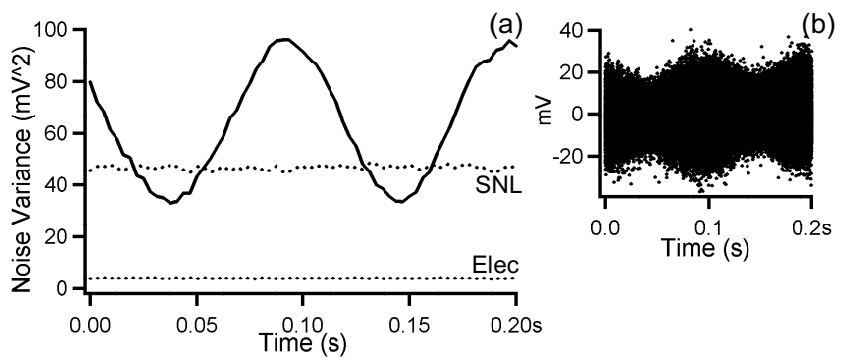

FIG. 4: Squeezed vacuum homodyne measurements in the time domain. Fig.(a) shows the noise variance (plotted in a linear scale and computed over blocks of 2,500 samples) while linearly scanning the LO phase, together with the shot noise level (SNL) and the electronic noise level. Fig(b) displays the corresponding recorded noise pulses.

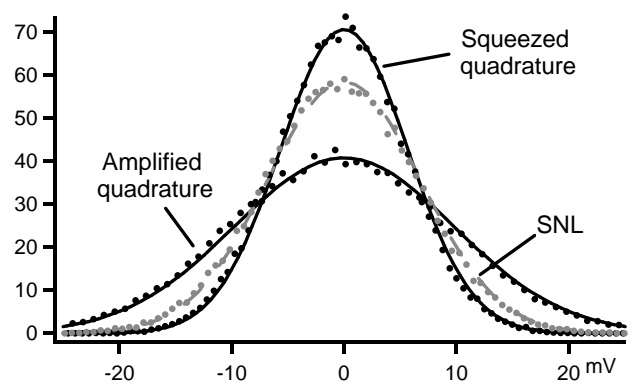

FIG. 5: Measured and theoretical pulse distributions for the anti-squeezed and squeezed quadratures. The squeezed quadrature variance is $1.87 \mathrm{~dB}$ below SNL, while the amplified quadrature variance lies $3.32 \mathrm{~dB}$ above SNL. oscillator phase. As expected for squeezed states, the measured noise variance passes below the shot noise level (SNL) for some phase values. The measured and theoretical Gaussian distributions corresponding to the squeezed and anti-squeezed quadratures are plotted on Fig. 5

The measured squeezing variance (with no correction) lies $-1.87 \pm 0.06 \mathrm{~dB}$ below the shot noise level, while the corresponding anti-squeezing variance is $3.32 \pm 0.04 \mathrm{~dB}$ above SNL. These results are in good agreement with the inferred levels $-1.92 \pm 0.06 \mathrm{~dB}$ and $3.32 \pm 0.06 \mathrm{~dB}$, obtained from the measured classical parametric gains of a probe beam $(0.53 \pm 0.01$ and $2.51 \pm 0.05)$ together with our evaluation of the overall detection efficiency $\eta$. The procedure to measure the detection efficiency is well established from squeezing experiments [2], and it can be cross-checked by comparing the classical parametric gain and the measured degree of squeezing. We note the overall detection efficiency $\eta=\eta_{T} \eta_{H}^{2} \eta_{D}=0.76 \pm 0.01$, where the overall transmission $\eta_{T}=0.92$, the mode-matching visibility $\eta_{H}=0.935$, and the detectors efficiency (Hamamatsu S3883) $\eta_{D}=0.945$ are independantly measured. The squeezed state measurements showed no sign of temporal mismatch with the LO pulse.

In conclusion, pulsed squeezed states have been easily and efficiently generated using ultrafast frequency conversions in thin $\mathrm{KNbO}_{3}$ crystals, leading up to $1.87 \mathrm{~dB}$ reduction in quadrature variance. A pulsed homodyne detection has been used to sample the quantum properties of each individual femtosecond pulse in the time domain, providing all the basic ressources for future quantum communication protocols using squeezed states.

We thank F. Grosshans for his contribution to the early steps of the experiment. This work was supported by the European IST/FET/QIPC program, and by the French programs "ACI Photonique" and "ASTRE". Email : jerome.wenger@iota.u-psud.fr
[1] S.L. Braunstein and A.K. Pati, Quantum Information with Continuous Variables, (Kluwer Academic, Dordrecht, 2003).

[2] R.E. Slusher, P. Grangier, A. LaPorta, B. Yurke and M.J. Potasek, Phys. Rev. Lett. 592566 (1987).

[3] P. Kumar, O. Aytur and J. Huang, Phys. Rev. Lett. 64, 1015 (1990).

[4] C. Kim and P. Kumar, Phys. Rev. Lett. 73, 1605 (1994).

[5] M.E. Anderson, M. Beck, M.G. Raymer and J.D. Bierlein, Opt. Lett. 20, 620 (1995).

[6] E.M. Daly, A.S. Bell, E. Riis and A.I. Ferguson, Phys. Rev. A 57, 3127 (1998).

[7] D.T. Smithey, M. Beck, M.G. Raymer and A. Faridani, Phys. Rev. Lett. 70, 1244 (1993).

[8] A.M. Weiner, A.M. Kan'an and D.E. Leaird, Opt. Lett. 23, 1441 (1998).
[9] D. Guzun, Y.Q. Li and M. Xiao, Opt. Commun. 180, 367 (2000).

[10] F. Grosshans, G. Van Assche, J. Wenger, R. Brouri, N.J. Cerf and Ph. Grangier, Nature 421, 238 (2003).

[11] H. Hansen, T. Aichele, C. Hettich, P. Lodahl, A.I. Lvovsky, J. Mlynek and S. Schiller, Opt. Lett. 26, 1714 (2001).

[12] H. Mabuchi, E.S. Polzik and H.J. Kimble, J. Opt. Soc. Am. B 11, 2023 (1994).

[13] A.D. Ludlow, H.M. Nelson, and S.D. Bergeson, J. Opt. Soc. Am. B 18, 1813 (2001).

[14] R.G. Reeves, M.G. Jani, B. Jassemnejad, R.C. Powell, G.J. Mizell and W. Fay, Phys. Rev. B 43, 71, (1991).

[15] A. Laporta and R.E. Slusher, Phys. Rev. A 442013 (1991). 